\title{
Energy Consumption Analysis of Video Streaming to Android Mobile Devices
}

\author{
Ramona Trestian ${ }^{1}$, Arghir-Nicolae Moldovan ${ }^{2}$, Olga Ormond ${ }^{1}$, Gabriel-Miro Muntean ${ }^{1}$, Member, IEEE \\ ${ }^{1}$ Performance Engineering Laboratory, School of Electronic \\ Engineering, Dublin City University, Ireland \\ \{ramona, ormondo, munteang\}@eeng.dcu.ie \\ ${ }^{2}$ School of Computing, National College of Ireland, \\ Dublin, Ireland \\ amoldovan@stundet.ncirl.ie
}

\begin{abstract}
Energy conservation has become a critical issue around the world. In smart phones, battery power capabilities are not keeping up with the advances in other technologies (e.g., processing and memory) and are rapidly becoming a concern, especially in view of the growth in usage of energy-hungry mobile multimedia streaming. The deficiency in battery power and the need for reduced energy consumption provides motivation for researchers to develop energy efficient techniques in order to manage the power consumption in next-generation wireless networks. As there is little analysis in the literature on the relationship between the wireless environment and the mobile device energy consumption, this paper investigates the impact of network-related factors (e.g., network load and signal quality level) on the power consumption of the mobile device in the context of video delivery. This paper analyzes the energy consumption of an Android device and the efficiency of the system in several scenarios while performing video delivery (over UDP or TCP) on an IEEE 802.11g network. The results show that the network load and the signal quality level have a combined significant impact on the energy consumption. This analysis can be further used when proposing energy efficient adaptive multimedia and handover mechanisms.

Keywords—mobile device energy consumption; adaptive multimedia streaming; wireless networks.
\end{abstract}

\section{INTRODUCTION}

Lately, smart mobile computing devices have become increasingly affordable and powerful, leading to a significant growth in both the number of advanced mobile users and their bandwidth demands. According to Cisco, the mobile data traffic generated by these high-end devices is expected to reach 6.3 exabytes per month by 2015 , increasing 26 -fold from the 2010 level of data use [1]. Driven by the growing popularity of websites and applications including video-sharing (e.g., YouTube, Vimeo, etc.), social networks (e.g. Facebook, Twitter, etc.), mobile IPTV, video-conferencing, etc., videobased applications have seen the highest growth rate of any application category. Video content is expected to account for two-thirds of global mobile data traffic by 2015 [1].

The continued growth of video content creates challenges for network service providers in ensuring a seamless multimedia experience at high quality levels to the end-user. One possible solution to deal with this explosion of mobile broadband data is to use Wi-Fi offload. WLAN networks have had an important impact in the area of mobile communications and their use has grown significantly in recent years (e.g., extended coverage, low-latency, power-efficient connection, reduced loads, etc.).

This work was supported in part by Enterprise Ireland Proof of Concept Fund, Science Foundation Ireland Research Frontiers Programme, Dublin City University, and IRCSET (Irish Research Council for Science, Engineering \& Technology) Embark Postgraduate Scholarship Scheme.
The Wi-Fi offload solution is already adopted by many service providers, (e.g., Deutsche Telekom and iPass launched WiFi Mobilize $^{1}$ ). This solution enables transfer of some traffic from the core cellular network to $\mathrm{WiFi}$ at peak times. Thus, users can avail of a wider service offering. However, the overall experience is still far from optimal as providing high quality mobile video services with QoS (Quality of Service) provisioning over resource-constrained wireless networks remains a challenge. Moreover user mobility, as well as the heterogeneity of mobile devices (e.g., different operating systems, display size, CPU capabilities, battery limitations, etc.), and the wide range of the video-centric applications (e.g., VoD (Video On Demand), video games, video conferences, etc.), opens up the demand for user-centric solutions that adapt the application to the underlying network conditions and device characteristics. Content adaptation solutions have the potential to increase users QoE (Quality of Experience) [2] and existing technologies (e.g., Apple HTTP Live Streaming, Microsoft IIS Smooth Streaming, Adobe Dynamic Streaming) can be used to adapt the multimedia content streamed to mobile devices over wireless networks. A stream-switching technique is employed, in which a server stores the video content at different quality levels and switches between the streams based on client feedback (e.g., available bandwidth) dynamically maintaining the user perceived quality at high levels.

In terms of energy conservation, ICT (Information and Communications Technologies) are seen as part of the solution in order to reduce the carbon footprints, but ICT itself needs to become more energy efficient. EU Commission is pushing for ICT to reduce its own carbon footprint by $20 \%$ by 2015 . This makes the understanding of power consumption to be one of the key challenges in the next generation mobile multimedia networks for efficient power management solutions. Thus, the battery life of the mobile device is the key component that consumers and producers alike care most about. Handsets are used as mobile work and entertainment centres, for communications, listening to music/radio, taking photos, GPS services, playing games, using any of the available 500,000 mobile app ${ }^{2}$ on the market, and for multimedia playback /streaming. This places additional strain on a battery that users expect to last at least a full day without recharging. Moreover, for the latest mobile devices (e.g. iPhone 4), which merely last for several hours of intense usage (e.g., video streaming) [3], the short battery life represents one of the biggest contributors to users dissatisfaction [4]. In the absence of battery improvements suitable to meet the growing demand for power-

\footnotetext{
${ }^{1} \mathrm{http}: / /$ www.telekom-icss.com/dtag/cms/content/ICSS/en/1508330 www.appmodo.com
} 
hungry applications, mobile users require better power and battery management techniques to increase their battery performance. These energy-efficient techniques should manage and reduce power consumption while still meeting user's quality of experience expectations.

This paper investigates the relationship between the wireless environment and the energy consumption of the mobile device in the context of video delivery. It represents an in-depth study on how the wireless link quality and the network load impact the energy consumption of an Android device while performing on-demand streaming over an IEEE $802.11 \mathrm{~g}$ network. The study offers a better understanding of the device's energy consumption and demonstrates the necessity of considering network-related parameters (e.g., link quality, network load) when designing energy-efficient wireless video transmission schemes. It also highlights energy saving benefits brought by the use of an adaptive multimedia mechanism.

\section{RELATED WORKS}

The increase in popularity of the new smart devices and their applications, has determined a trend towards mobile users demanding more interactive and personalized multimedia services with high performance. It is known that real-time applications (e.g., multimedia-based) have strict QoS requirements, but they are also the most power-consuming ${ }^{3}$. In this context, one of the main impediments of progress is the battery lifetime of the mobile device.

Energy conservation has become a critical issue around the world and presents motivation for researchers to propose and develop energy efficient techniques in order to manage the power consumption in next-generation wireless multimedia networks. Zhang et al. in [5] present a survey on the recent major advances in power-aware multimedia. The main focus of the survey is on video coding and video delivery. The authors identify the main challenges that come when designing energy efficient mobile multimedia communication devices, as: 1) real-time multimedia is delay-sensitive and bandwidth-intense making it also the most power consuming application, 2) the radio frequency environment is changing dynamically over time and space, 3) the diversity of mobile devices and their capabilities, 4) the video quality does not present a linear increase with the increase in complexity, and 5) the battery discharge behavior is nonlinear. The authors conclude that due to the dynamics involved, enabling power-aware mobile multimedia is extremely challenging. Many tradeoffs are involved in the process, for example using high compression techniques to reduce the amount of data to be transmitted and therefore the energy involved in data delivery, but higher compression involves higher computation both at the client and the server, and therefore increased battery usage.

Rice et. al in [6] present a measurement framework that produces fine-grained traces of mobile device power consumption. The framework is then used to analyze and decompose the power consumption of two Android 1.5-based mobile devices into independent parts. The results show that the idle power consumption of the mobile device is higher in a $2 \mathrm{G}$ network than when connected to a $3 \mathrm{G}$ or a WiFi network.

\footnotetext{
${ }^{3}$ www.wallstreetandtech.com
}

Vallina-Rodriguez et al. [7] perform a study on collecting usage data of 18 Android OS users during a 2 weeks period (Feb. 2010) in order to understand the resource management and battery consumption pattern. The information collected from the mobile devices covers a wide range of parameters, more than 20 (e.g., CPU load, battery level, network type, network traffic, GPS status, etc.) being updated at every 10 seconds. The study shows the importance of contextual information when designing energy efficient algorithms. For example, by identifying where and when some resources are in high demand $(50 \%$ of their time the users were subscribed to their top three most common base stations) a more energy efficient resource management can be proposed that uses this information. The context information (time, history, network conditions, device motion) is also used in [8] by Rahmati et al. in order to estimate current and future network conditions and automatically select the most energy efficient network $(802.11 \mathrm{~b}$ or GSM/EDGE). The authors collected usage information from 14 users (HTC Wizard Pocket PC, HTC Tornado, and HP iPAQ hw6925 phones) during a 6 months period (Sept. $2006-$ Feb. 2007). The authors argue that by using the context-based interface selection mechanism the average battery lifetime of the mobile device can reach $35 \%$ increase comparing with the case of using the cellular interface only.

A study on the energy consumption of YouTube in mobile devices was carried out by Xiao et al. [9]. The authors measured the energy consumption of a Nokia S60 mobile phone for three different use cases (progressive download, download-and-play, and local playback) and for two access network technologies (WCDMA and WLAN). Even though the results show that the WCDMA network consumes more energy than WLAN, they do not consider the impact of fluctuating network bandwidth nor the quality of the video.

Perrucci et al. [10] investigate the energy consumption of a Nokia N95 while performing VoIP. The authors propose the use of a lower energy consumption interface (e.g., GSM) as a signaling channel to wake up the WLAN interface and run the VoIP service. The authors argue that by using the wake-up signals the energy consumption can be reduced significantly in a VoIP scenario. The use of sleep and wake-up schedules is used by Namboodiri et al. [11] for energy saving during VoIP calls. The authors propose a GreenCall algorithm that keeps the WLAN interface of a laptop in sleep mode for significant periods during the VoIP calls. The maximum delay that a user can tolerate during a call is used to compute the sleep periods.

Despite the amount of research done in the area of energy conservation, not much focus has been placed on the impact of the multimedia communication environment (e.g., link quality, location, technology, network load, etc.) on the energy consumption. In this paper we conducted a measurement analysis with the main goals:

- Understanding the energy-quality tradeoff ;

- Understanding the impact of the transport protocol (TCP or UDP) on the energy consumption for VoD over WLAN;

- Understanding the impact of the link quality on the energy consumption for VoD over WLAN;

- Understanding the impact of the network load on the energy consumption for VoD over WLAN;

- Understanding the impact of both link quality and network load on the energy consumption for VoD over WLAN; 


\section{EXPERIMENTAL TEST-BED SETUP}

This section presents the measurement environment setup, as illustrated in Fig. 1, consisting of: a Wireless-G Router, a Traffic Generator, a Multimedia Server, a Network Monitor, an Android Mobile Device, and a Power Consumption Monitor.

\section{A. Wireless Network Environment}

A Belkin N Wireless Router was configured for IEEE $802.11 \mathrm{~g}$ mode, running on channel 6 (freq. $2.437 \mathrm{GHz}$ ), with no other local networks running on the same or adjacent channel. Wi-Spy DBx USB spectrum analyzer from MetaGeek ${ }^{4}$ together with the accompanying Chanalyzer 4 software (running on the Network Monitor station), were used for monitoring the surrounding wireless networks and interference levels.

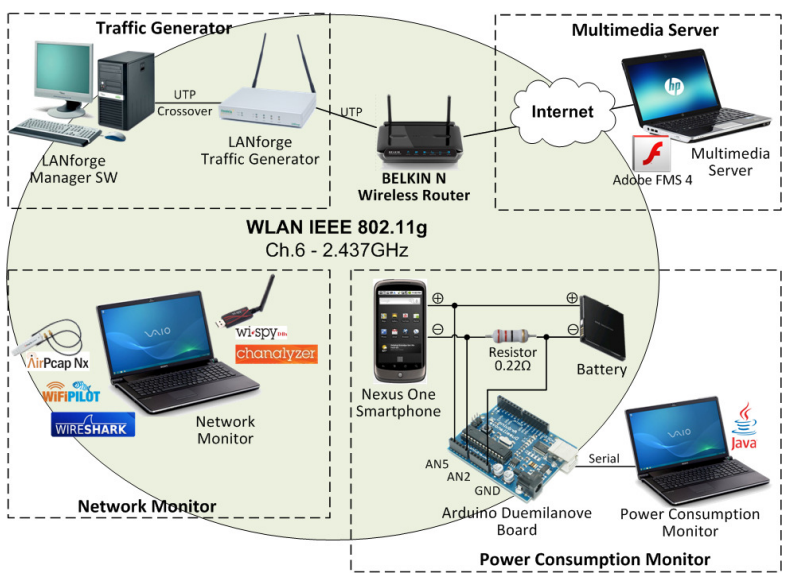

Figure 1. Experimental Test-bed Setup: Traffic Generator, Multimedia Server, Network Monitor and Power Consumption Monitor

To reduce interference, the tests were run in the basement of a building where a lower number of wireless networks were within range. Fig. 2 illustrates the $2.4 \mathrm{GHz}$ band in two situations: when no traffic is generated in the "Test" network, and when the network is loaded. As shown in Fig. 2, the other wireless networks in range are running on different (and nonadjacent) channels, so interference is kept at a minimum.

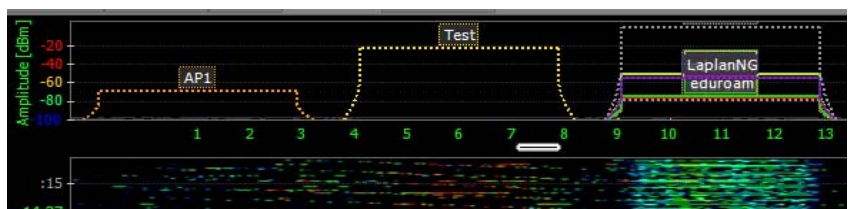

(a)

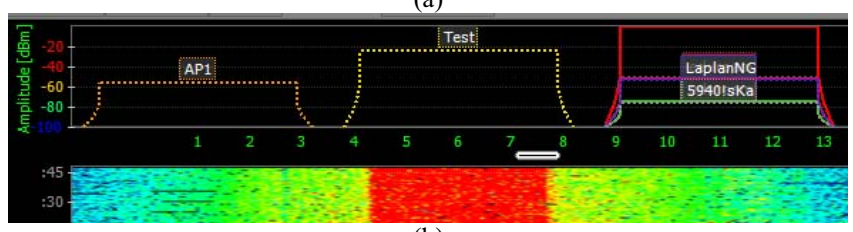

(b)

Figure 2. Chanalyzer 4 screenshots illustrating the wireless environment: (a) no traffic generated in the test network; (b) the test network is loaded with 20$21 \mathrm{Mbps}$ of background traffic.

In order to better understand what exactly is happening in the network, the traffic was captured with the help of AirPcap Nx (from MetaGeek) that includes WireShark 1.4.8 and Wifi

\footnotetext{
${ }^{4}$ www.metageek.net
}

Pilot 2.4 software (running on the Network Monitor station). The goal of the network traffic analysis is: to monitor the ondemand video streaming (e.g. received throughput, retransmissions vs. normal traffic, etc.), and to double-check that the background traffic is generated properly by the virtual stations created using the traffic generator.

\section{B. Background Traffic Generation}

Background traffic was generated in order to assess the impact of network load on the mobile device energy consumption. The traffic generator used was a CT520 LANforge-WiFIRE $802.11 \mathrm{a} / \mathrm{b} / \mathrm{g}$ from Candela Technologies ${ }^{5}$, which enables creation of up to 32 virtual stations. The background traffic was selected based on the traffic estimations provided by Cisco in [1] and by Plum Consulting ${ }^{6}$ in a report for Ericsson and Qualcomm. According to them, over the next five years the ratio of downlink (DL) to uplink (UL) traffic will rise to $10: 1$, while the video traffic is expected to reach $66 \%$ of the total mobile traffic. Thus, the choice on the background traffic is based on the traffic forecast for 2015 and is listed in Table I. In this way we create a more realistic environment and we can analyze what to expect in terms of network conditions over the next five years.

In order to load the IEEE 802.11g network, we first measured the available bandwidth using Jperf ${ }^{7}$ in Server mode at the Server side, together with Iperf for Android in Client mode on the mobile device. Iperf measures the available bandwidth between two end points by generating probe traffic into the network. In order to obtain accurate results, we took ten Iperf readings at $30-50$ s intervals between readings and computed the average available bandwidth which was in the range of $21-23 \mathrm{Mbps}$. Based on these measurements we selected the traffic load of the network in the range of 20$21 \mathrm{Mbps}$ and the traffic type according to Table I. The number of wireless clients generating background traffic is in the range of 25-28 clients, located near the AP with the Signal Strength (SS) between $-25 \mathrm{dBm}$ and $-35 \mathrm{dBm}$. Video here represents traditional video traffic over UDP with data rates $0.25-2 \mathrm{Mbps}$ and a packet size of 1514 bytes. The other traffic represents web-browsing/e-mail, and file sharing, etc. it is TCP traffic with data rates $0.25-1 \mathrm{Mbps}$ and packet size in the range 300-1514bytes.

TABLE I. BACKGROUND TRAFFIC

\begin{tabular}{|c|c|c|c|}
\hline Type & \% Traffic Cisco 2015 & \% downlink & $\%$ uplink \\
\hline Video & $66 \%$ & $98 \%$ & $2 \%$ \\
\hline Other & $34 \%$ & $76 \%$ & $24 \%$ \\
\hline
\end{tabular}

The traffic generated by the Android Mobile Device, falls into the downlink video traffic category. As the corresponding traffic data rate changes according to the video quality level, we change the background traffic in order to maintain the same percentage $(66 \%)$ in all scenarios.

\section{Multimedia Encoding and Streaming}

The Blender Foundation's 10 minute long Big Buck Bunny ${ }^{8}$ animated clip was used for testing. A high quality version of

\footnotetext{
5 www.candelatech.com

${ }^{6}$ www.plumconsulting.co.uk

7 www.iperf.sourceforge.net

${ }^{8}$ Big Buck Bunny.
} 
the clip was transcoded at five different quality levels, following recommendations for encoding clips for multibitrate adaptive streaming ${ }^{9}$. The encoding characteristics of the five test sequences are presented in Table II. H.264/Mpeg-4 AVC video compression and AAC audio compression were used together with the mp4 format. The highest resolution was selected as $800 \times 448$ pixels to fit the screen resolution of the Android device $(800 \times 480$ pixels), while maintaining the original aspect ratio of the multimedia clip (16:9). The video frame rate was decreased from 30fps (QL1) to 10fps (QL5). The overall bitrate was decreased by half between consecutive quality levels starting from $1920 \mathrm{Kbps}$ (QL1). The test sequences were streamed to the Android device over both TCP and UDP transport protocols. Adobe Flash Media Server $4^{10}$ was used for streaming the videos using the proprietary application level streaming protocols RTMP (TCP) and RTMFP (UDP). The streams were embedded in web pages and were played back on the device using Adobe 10.2 Flash Player inside the Android native web browser. The video playout is scaled to the device screen resolution.

TABLE II. EnCoding SetTings For THE Multimedia TeSt SEQuenceS

\begin{tabular}{|c|c|c|c|c|c|}
\hline $\begin{array}{c}\text { Quality } \\
\text { Level }\end{array}$ & $\begin{array}{l}\text { Video } \\
\text { Codec }\end{array}$ & $\begin{array}{c}\text { Overall Bitrate } \\
{[\mathrm{Kbps}]}\end{array}$ & $\begin{array}{l}\text { Resolution } \\
\text { [pixels] }\end{array}$ & $\begin{array}{c}\text { Frame Rate } \\
\text { [fps] }\end{array}$ & $\begin{array}{l}\text { Audio } \\
\text { Codec }\end{array}$ \\
\hline QL1 & \multirow{5}{*}{$\begin{array}{c}\text { H.264/ } \\
\text { MPEG-4 } \\
\text { AVC } \\
\text { Baseline } \\
\text { Profile }\end{array}$} & 1920 & $800 \times 448$ & 30 & \multirow{5}{*}{$\begin{array}{c}\text { AAC } \\
25 \\
\mathrm{Kbps} \\
8 \mathrm{KHz}\end{array}$} \\
\hline QL2 & & 960 & $512 \times 288$ & 25 & \\
\hline QL3 & & 480 & $320 \times 176$ & 20 & \\
\hline QL4 & & 240 & $320 \times 176$ & 15 & \\
\hline QL5 & & 120 & $320 \times 176$ & 10 & \\
\hline
\end{tabular}

\section{Objective Quality Assessement}

Since video quality is an important aspect of multimedia delivery, Peak Signal-to-Noise Ratio (PSNR), a full-reference objective metric, was measured in order to estimate the human perceived visual quality offered by the five encoding settings used. MSU Video Quality Measurement Tool ${ }^{11}$ software was used for computing the objective quality values. PSNR is measured by comparing the quality of the degraded versions (QL2 to QL5) with respect to that of the highest quality sequence (QL1). Since this is done on a pixel-by-pixel basis, all the clips were scaled to the same video resolution and video frame rate. Although employing the scaling process is not ideal, by computing PSNR one gets a good idea of the human perceived quality levels for these video sequences.

\section{E. Subjective Quality Assessement}

Since objective metrics do not always correlate with the subjective scores, a subjective study was also conducted in order to assess how human subjects perceive the quality of the multimedia clip encoded at five quality levels previously selected (see Table II). Four 20 seconds long sequences with different spatial and temporal characteristics were extracted from the original 10 minute long clip. Representative frames of the four sequences are presented in Fig. 3. In case of sequence $\mathrm{A}$ the camera pans slowly over a natural landscape scene, thus the sequence presents a medium level of spatial information and a low level of temporal information. Sequence B is the most complex to encode. It presents fast changing scenes with dynamic elements as well as characters,

\footnotetext{
${ }^{9}$ Smooth Streaming Multi-Bitrate Calculator

${ }^{10}$ Adobe Flash Media Server.

${ }^{11}$ MSU Video Quality Measurement Tool
}

thus having the highest levels of spatial and temporal complexity. Sequence C is especially difficult to encode at low resolutions due to the small moving details represented by the closing credits. Sequence D presents two characters, from which only one is slowly moving across the scene, on a static background. Therefore the scene has the lowest level of spatial information. Each of the four sequences was encoded at the five quality levels, resulting in a total number of 20 test sequences for the subjective study. The test sequences were played locally in full screen on the Nexus One Android device, and displayed in a random order, maintaining similar testing conditions for all the participants. Standard recommendations for assessing the visual quality of multimedia applications were followed as in [12]. The Absolute Category Rating (ACR) [12] method was used, where the subjects had to individually rate the quality of each sequence on a 5-point scale (e.g., 1-Bad, 2-Poor, 3-Fair, 4Good, 5-Excellent). A number of $16(\mathrm{M}=10, \mathrm{~F}=6)$ non-expert subjects with ages between 22 and 45 years old $(A V G=28$, STDEV=6) participated in the study. All the subjects have reported that they had normal vision or had corrected to normal vision (they were wearing glasses).

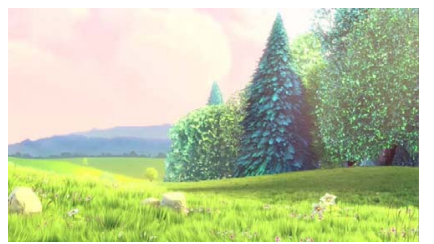

a) Sequence $\mathrm{A}$

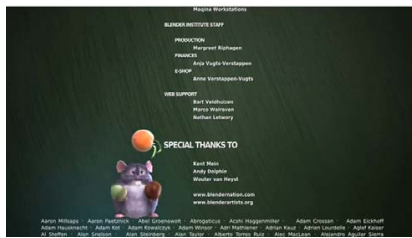

c) Sequence C

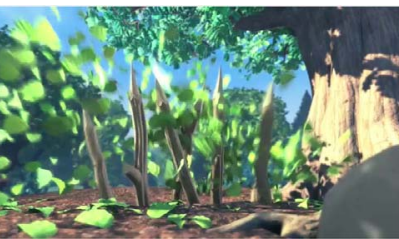

b) Sequence B

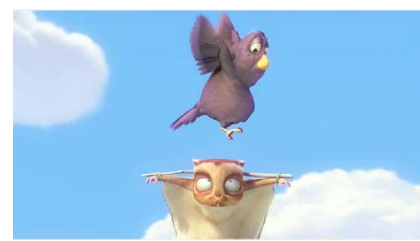

d) Sequence D

\section{F. Energy Measurement}

A HTC Google Nexus One smartphone running Android 2.3.4 was selected as the client Mobile Device. Android Mobile Devices have rapidly grown in popularity over the recent years, reaching nearly $50 \%$ of the global smartphone market by the latest estimates [13]. As opposed to other smartphones, in particular to iPhone, it has the advantage of a user replaceable battery. Having access to the battery contacts, the device power consumption can be measured using hardware equipment, thus having more accurate results than using locally installed software.

An Arduino Duemilanove board ${ }^{12}$ was used for measuring the battery voltage as well as the voltage drop on a shunt resistor inserted in between the device and the battery, in order to determine the current (see Fig. 1). A Java application, running on the Power Consumption Monitor station, was used to compute the device power consumption based on the voltage values sent by the Arduino board. The values were saved at $1 \mathrm{~Hz}$ frequency.

\footnotetext{
${ }^{12}$ Arduino Duemilanove Board
} 


\section{TEST SCENARIOS}

In order to study how the signal quality (distance from AP) and network load impact on the power consumption of an Android Mobile Device, we considered four scenarios as illustrated in Fig. 4 and described below. In all considered scenarios the Multimedia Server stores five ten-minute clips, each clip corresponding to a different quality level. The clips are streamed sequentially to the Android Mobile Device over either of two transport protocols (UDP and TCP).

- Scenario 1 - considers the case of a mobile user, located near the AP (approximately $1 \mathrm{~m}$ away), without any background traffic in the network. The SS varies between [-48dBm, $-52 \mathrm{dBm}]$.

- Scenario 2 - the mobile user is located in a poor area with the SS varying between $[-78 \mathrm{dBm},-82 \mathrm{dBm}]$. The tests were run without any background traffic in the network in order to study the impact of the link quality on the energy consumption of the mobile device.

- Scenario 3 - similar to the first, except that background traffic was added in order to load the network, and study the impact of the network load on the energy consumption of the mobile device. LANforge traffic generator was used to create 25-28 virtual wireless stations, each generating traffic as previously explained in Section III.B.

- Scenario 4 - similar to scenario 2 except that background traffic was added as in scenario 3 , in order to study the impact of both poor link quality and network load, on the energy consumption of the mobile device.

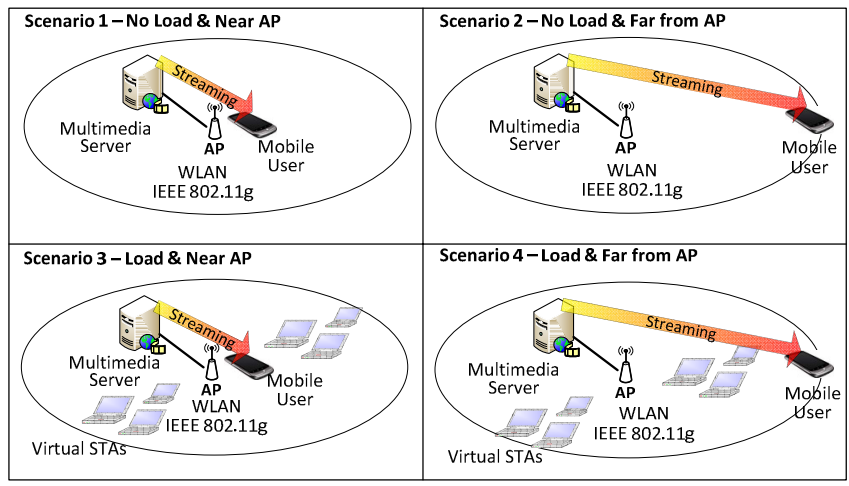

Figure 4. Considered Test Scenarios

\section{RESULTS AND ANALYSIS}

For each considered scenario and for each of the quality levels we repeated the test three times (a total of 195 tests were carried out) and computed the average values. These values are further used throughout this paper for analysis of the results and discussions.

1) Impact of the Video Quality Levels on Human Perceived Visual Quality with Local Video Playback

To assess the user perceived quality of the five quality levels, the subjects were asked to view 20 test sequences and rate their overall quality on a 1-5 scale (bad to excellent). For each sequence, the mean value represented by the Mean Opinion Score (MOS), and the standard deviation (STDEV) of the statistical distribution of the assessment grades were computed. The results of the subjective study are presented in
Fig. 5. All the sequences corresponding to QL1-QL3, scored above 4 (Good), with eight of them scoring above 4.5 (Excellent). Out of the eight test sequences corresponding to QL4 and QL5, four scored above 3.5 (Good) on average, while the other two below 3.5 but above 2.5 (Fair) on average. On average across the four test sequences, two quality levels scored Excellent (MOS_QL1=4.84 and MOS_QL2=4.63), two scored Good (MOS QL3 $=4.33$ and MOS QL4=3.70) and one Fair (MOS_QL5 $=\overline{3} .38$ ). The average standard deviation values, shown in Fig. $5 \mathrm{~b}$ increase as the video quality decreases (AVG_STDEV_QL1 $=0.35$ to AVG_STDEV_QL5 $=0.90$ ). The Pearson correlation further indicates that there is decreasing relationship between the MOS and STDEV values $(\mathrm{r}=-0.846)$, thus the ratings across participants tend to have a higher variation, for the clips with lower perceived quality.
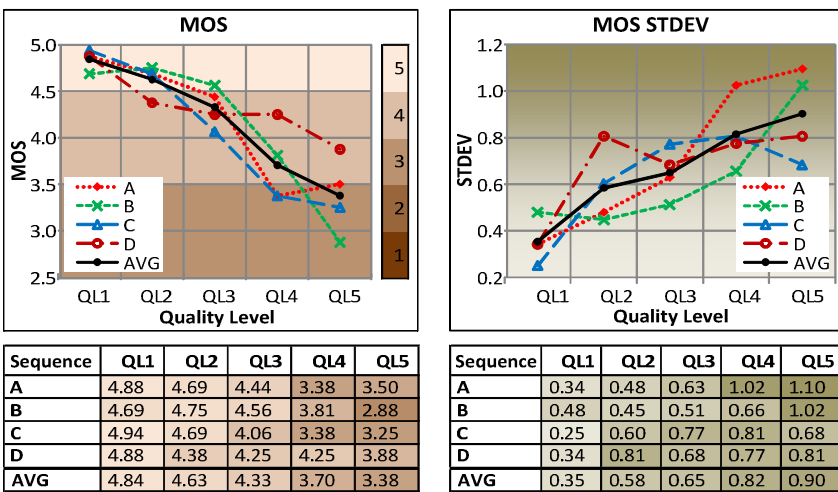

a) $\mathrm{MOS}$

Figure 5. Subjective Assessment Results

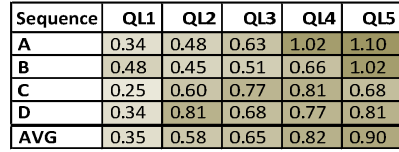

MOS STDEV

2) Impact of the Video Quality Levels on Energy Consumption of Local Playback

In order to study how much energy can be conserved by changing the quality level of the video, we performed local video playback of each quality level. All the tests were performed with the same minimal background activities on the mobile device, with all the wireless connectivity interfaces disabled (Airplane Mode) and the power save mode turned off. The results are illustrated in Table III. The battery discharge and the battery life values were estimated using the equations as expressed below:

\section{Discharge $[\mathrm{mAh}]=$ Avg. Energy $[J] * 1000 /(3.7[\mathrm{~V}] * 3600[\mathrm{~s}])$ \\ Battery Life $[\mathrm{hrs}]=1330[\mathrm{mAh}] * 3.7[\mathrm{~V}] /$ Avg. Power $[\mathrm{mW}]$}

where, $3.7 \mathrm{~V}$ and $1330 \mathrm{mAh}$ represent the nominal battery voltage and battery capacity respectively. The battery discharge represents the total charge drawn from the battery during the clip playback. The Battery Life represents the amount of time the fully charged battery will take to discharge while playing a certain quality level. For example, if only QL1 videos are played, the device has an estimated battery life of 4 hours, while by choosing to play only QL5 videos, the battery life is doubled. The results show that by decreasing the video quality level we can achieve energy savings. Switching from QL4 to QL5 provides a low saving of $4.5 \%$ for a corresponding MOS decrease from Good to Fair. However switching from QL1 to QL3 provides a $44.8 \%$ energy saving for a MOS decrease from Excellent to Good, while a switch from QL1 to QL2 offers 34\% energy savings at no change in MOS. 
TABLE III. RESULTS FOR LOCAL PLAYBACK

\begin{tabular}{|c|c|c|c|c|c|c|c|}
\hline $\begin{array}{c}\text { Quality } \\
\text { Level }\end{array}$ & $\begin{array}{c}\text { Avg. } \\
\text { Energy [J] }\end{array}$ & $\begin{array}{c}\text { STDEV } \\
\text { Energy }\end{array}$ & $\begin{array}{c}\text { Avg. } \\
\text { Power } \\
{[\mathbf{m W}]}\end{array}$ & $\begin{array}{c}\text { Discharge } \\
{[\mathbf{m A h}]}\end{array}$ & $\begin{array}{c}\text { Battery } \\
\text { Life }[\mathbf{h r s}]\end{array}$ & $\begin{array}{c}\text { PSNR } \\
{[\mathbf{d B}]}\end{array}$ & $\begin{array}{c}\text { Subjective } \\
\text { MOS }\end{array}$ \\
\hline \hline QL1 & 712 & 3.28 & 1196 & 53 & 4.11 & - & 4.84 \\
\hline QL2 & 470 & 1.18 & 788 & 35 & 6.24 & 47 & 4.63 \\
\hline QL3 & 393 & 1.06 & 658 & 29 & 7.48 & 41 & 4.33 \\
\hline QL4 & 374 & 1.03 & 627 & 28 & 7.85 & 36 & 3.70 \\
\hline QL5 & 357 & 4.15 & 598 & 27 & 8.23 & 31 & 3.38 \\
\hline
\end{tabular}

3) Impact of the Video Quality Levels on Energy Consumption while Performing VoD

Considering Scenario 1, with the mobile device located near the AP and without background traffic, we measure the energy consumption while performing VoD over UDP. The difference between these results and the local playback gives us an overview of the energy consumption over the wireless network. The impact of the wireless interface on the energy consumption is illustrated in Fig. 6 and Table IV. The results show that by decreasing the video quality level with $\mathrm{VoD}$, we can achieve energy savings from $6.7 \%$ (for a QL1 to QL2 drop) up to $62.7 \%$ (for a QL1 to QL5 decrease) on the wireless interface only. Because the link is good quality and enough available bandwidth is provided for VoD, the playback is smooth and uninterrupted, maintaining the same user perceived quality and the same subjective MOS values as for local playback.

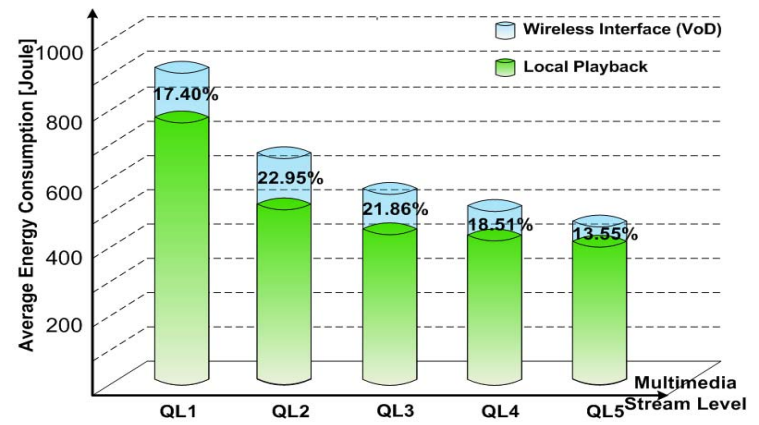

Figure 6. Avg. Energy Consumption of the Mobile Device for UDP VoD vs. Local Playback

4) Impact of the Transport Protocol on Energy Consumption while Performing VoD

TCP was built for reliable data transport offering fairness to users by dividing available resources in an almost equal manner. As TCP congestion control mechanisms can affect video streaming, the traditional method of transporting video is over UDP. However, nowadays the use of TCP has become ubiquitous for streaming video, and it has been adopted in combination with adaptive multimedia solutions (e.g., Apple HTTP live streaming, Move Networks, etc.).

Considering Scenario 1, we run the same five quality levels, keeping the same conditions and changing only the transport protocol (UDP or TCP). The results illustrated in Table IV show TCP is more energy efficient than UDP. Fig. 7 illustrates the difference in terms of energy consumption. For QL1, 13\% energy savings can be achieved on the wireless interface by transmitting over TCP rather than UDP. One of the possible reasons for which TCP performs better, is that its packet size distribution is 1280-2559 bytes, meaning larger, but fewer packets to be transmitted. On the other hand the UDP packet size distribution is lower, 640-1279 bytes, meaning more packets to be transmitted.

TABLE IV. SCENARIO 1 - UDP VS. TCP VOD

\begin{tabular}{|c|c|c|c|c|c|c|c|}
\hline & $\begin{array}{l}\text { Quality } \\
\text { Level }\end{array}$ & $\underset{\text { Avg. }}{\text { Energy }[\mathrm{J}]}$ & $\begin{array}{l}\text { STDEV } \\
\text { Energy }\end{array}$ & $\begin{array}{c}\text { Avg. } \\
\text { Power } \\
\text { [mW] }\end{array}$ & $\begin{array}{l}\text { Discharge } \\
\text { [mAh] }\end{array}$ & $\begin{array}{c}\text { Battery Life } \\
\text { [hrs] }\end{array}$ & $\begin{array}{c}\text { Avg. Th. } \\
\text { [Mbps] }\end{array}$ \\
\hline \multirow{5}{*}{$\hat{\mathrm{s}}$} & QL1 & 862 & 7.48 & 1445 & 65 & 3.41 & 2.07 \\
\hline & QL2 & 610 & 2.75 & 1022 & 46 & 4.82 & 1.05 \\
\hline & QL3 & 503 & 5.16 & 841 & 38 & 5.85 & 0.52 \\
\hline & QL4 & 459 & 2.43 & 764 & 34 & 6.44 & 0.26 \\
\hline & QL5 & 413 & 3.89 & 699 & 31 & 7.04 & 0.14 \\
\hline \multirow{5}{*}{ :ै } & QL1 & 842 & 2.11 & 1410 & 63 & 3.49 & 2.02 \\
\hline & QL2 & 567 & 3.43 & 953 & 43 & 5.16 & 1.00 \\
\hline & QL3 & 475 & 3.63 & 799 & 36 & 6.16 & 0.51 \\
\hline & QL4 & 434 & 3.63 & 726 & 33 & 6.78 & 0.26 \\
\hline & QL5 & 398 & 3.45 & 666 & 30 & 7.39 & 0.14 \\
\hline
\end{tabular}

The actual average throughput (Avg. Th.) received by the mobile device on the wireless network, was captured with Wireshark and listed in Table IV. As seen, the required throughput for each quality level (Table II) is provided.

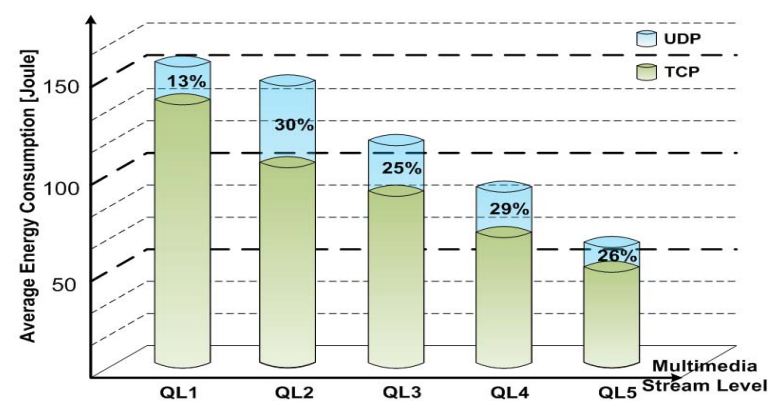

Figure 7. Scenario 1 - Avg. Energy Consumption for the Wireless Interface for UDP vs. TCP VoD

\section{5) Impact of the Link Quality on Energy Consumption}

In order to study the impact of the link quality on the energy consumption of the mobile device, we considered Scenario 1 and 2, when the user is located near the AP with good SS (-48 to $-53 \mathrm{dBm})$ and when the user is located in a poor SS area $(-78$ to $-82 \mathrm{dBm})$. In both scenarios no background traffic is considered, so SS is the only varying factor. The results are listed in Table IV and V. Fig. 8 illustrates the impact of the link quality on energy consumption for both transport protocols (UDP and TCP) by comparing the wireless interface energy consumption for Scenario 1 vs. Scenario 2.

TABLE V. SCENARIO 2 - UDP AND TCP VOD

\begin{tabular}{|c|c|c|c|c|c|c|c|}
\hline & $\begin{array}{c}\text { Quality } \\
\text { Level }\end{array}$ & $\underset{[\mathrm{J}]}{\text { Avg. Energy }}$ & $\begin{array}{l}\text { STDEV } \\
\text { Energy }\end{array}$ & $\begin{array}{l}\text { Avg. } \\
\text { Power } \\
\text { [mW] }\end{array}$ & $\begin{array}{c}\text { Discharge } \\
\text { [mAh] }\end{array}$ & $\begin{array}{c}\text { Battery Life } \\
{[\mathrm{hrs}]}\end{array}$ & $\begin{array}{l}\text { Avg. Th. } \\
\text { [Mbps] }\end{array}$ \\
\hline \multirow{5}{*}{ ลิ) } & QL1 & 875 & 3.74 & 1461 & 66 & 3.37 & 3.32 \\
\hline & QL2 & 628 & 7.86 & 1052 & 47 & 4.68 & 1.57 \\
\hline & QL3 & 512 & 5.05 & 857 & 38 & 5.74 & 0.59 \\
\hline & QL4 & 463 & 2.5 & 777 & 35 & 6.34 & 0.26 \\
\hline & QL5 & 420 & 2.7 & 704 & 32 & 6.99 & 0.13 \\
\hline \multirow{5}{*}{ บิ } & QL1 & 865 & "1.8 & 1448 & 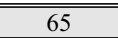 & 3.40 & 2.15 \\
\hline & QL2 & 586 & 3.23 & 982 & 44 & 5.01 & 0.98 \\
\hline & QL3 & 492 & 2.83 & 823 & 37 & 5.98 & 0.53 \\
\hline & QL4 & 446 & 2.66 & 746 & 33 & 6.60 & 0.32 \\
\hline & QL5 & 414 & 5.50 & 692 & 31 & 7.11 & 0.15 \\
\hline
\end{tabular}




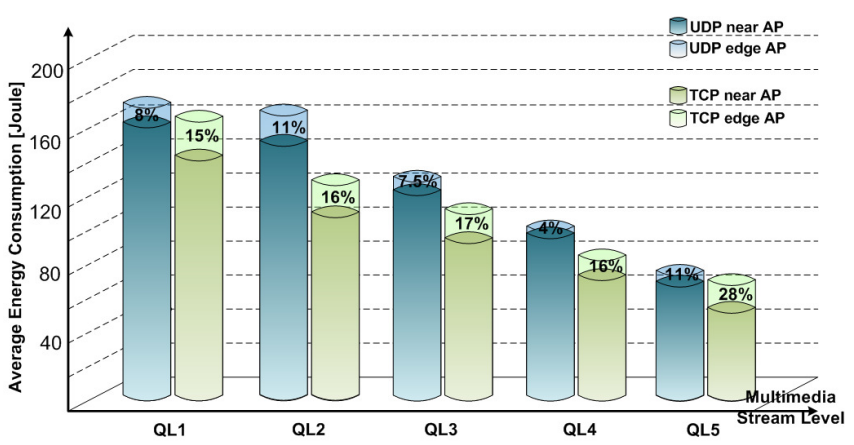

Figure 8. Scenario 1 vs. Scenario 2 Avg. Energy Consumption for the Wireless Interface for UDP vs. TCP VoD

As shown a poor SS has a higher impact on the TCP wireless interface energy consumption over UDP, with as high as $28 \%$ increase in energy usage for QL5 at the edge. The decrease in SS has a lesser impact on UDP, with as low as 4\% increase in energy for QL4 at the edge, up to an 11\% increase (QL2 and QL5). However, even in these conditions, TCP remains more energy efficient than UDP. The actual received throughput results meet the required throughput for each quality level (Table II) meaning smooth uninterrupted playback and maintained user perceived quality as for local playback.

\section{6) Impact of the Network Load on Energy Consumption}

By comparing Scenario 1 and 3 we can determine the impact of the network load on the energy consumption of the mobile device. Table VI presents the energy information and network related measurements captured by Wireshark.

TABLE VI. SCENARIO 3 - UDP AND TCP VOD

\begin{tabular}{|c|c|c|c|c|c|c|c|c|c|}
\hline & $\begin{array}{r}\text { Quality } \\
\text { Level }\end{array}$ & $\begin{array}{c}\text { Avg. } \\
\text { Energy } \\
{[J]}\end{array}$ & $\begin{array}{l}\text { STDEV } \\
\text { Energy }\end{array}$ & $\begin{array}{c}\text { Avg. } \\
\text { Power } \\
{[\mathrm{mW}]} \\
\end{array}$ & $\begin{array}{c}\text { Discharge } \\
\text { [mAh] }\end{array}$ & $\begin{array}{c}\text { Battery } \\
\text { Life } \\
\text { [hrs] }\end{array}$ & $\begin{array}{c}\text { Avg. } \\
\text { Th. } \\
\text { [Mbps] }\end{array}$ & $\begin{array}{c}\text { Avg. Ch. } \\
\text { Traffic } \\
\text { [Mbps] }\end{array}$ & $\begin{array}{l}\text { Retr. } \\
{[\%]}\end{array}$ \\
\hline \multirow{5}{*}{$\bar{E}$} & QL1 & 897 & 4.61 & 1489 & 67 & 3.30 & 2.27 & 24.32 & 3.82 \\
\hline & QL2 & 657 & 3.19 & 1102 & 49 & 4.47 & 1.18 & 25.12 & 7.98 \\
\hline & QL3 & 536 & 5.10 & 895 & 40 & 5.50 & 0.65 & 24.97 & 8.37 \\
\hline & QL4 & 466 & 3.24 & 779 & 35 & 6.32 & 0.36 & 24.90 & 5.61 \\
\hline & QL5 & 438 & 5.29 & 733 & 33 & 6.71 & 0.18 & 24.89 & 5.98 \\
\hline \multirow{5}{*}{ ర } & $\overline{\overline{\text { QL1 }}}$ & $\overline{885}$ & $4 \overline{4.56}$ & 1483 & $\bar{~} 66$ & 3.32 & 2.09 & 24.46 & $4 \quad 4.07$ \\
\hline & QL2 & 615 & 3.94 & 1030 & 46 & 4.78 & 1.06 & 24.66 & 4.79 \\
\hline & QL3 & 495 & 1.42 & 829 & 37 & 5.93 & 0.67 & 24.84 & 5.28 \\
\hline & QL4 & 462 & 6.54 & 774 & 35 & 6.36 & 0.35 & 24.18 & 9.1 \\
\hline & QL5 & 415 & 5.46 & 695 & 31 & 7.08 & 0.30 & 24.69 & 5.57 \\
\hline
\end{tabular}

The average received throughput (Avg. Th.) more than meets the requested throughput for each quality level. This means that even though with the high network load, every user receives their requested network resources. This is also shown by the average value of the overall channel traffic (Avg. Ch. Traffic). The payload of the overall network traffic was set as 20-21Mbps, but with network overhead it reaches $24-25 \mathrm{Mbps}$ (according to Wireshark).

Another important factor is the number of retransmissions (Retr.) that occur in the network. This value shows the relative number of the overall packets that were retransmitted vs. normal traffic, and is expressed as a percentage. Due to the high number of clients (26 in this case) that share the network, the competition for the network resources is high and this is reflected by the retransmissions value. Fig. 9 illustrates how this affects the energy consumption. Looking at QL2 transmission over UDP, it can be seen that when the network is loaded it consumes more or less the same energy as QL1.
This is due to network contention, as the overall retransmissions double compared with QL1. Also the average channel traffic presents an increase of $3.2 \%$ reflecting the increase in the resource competition.

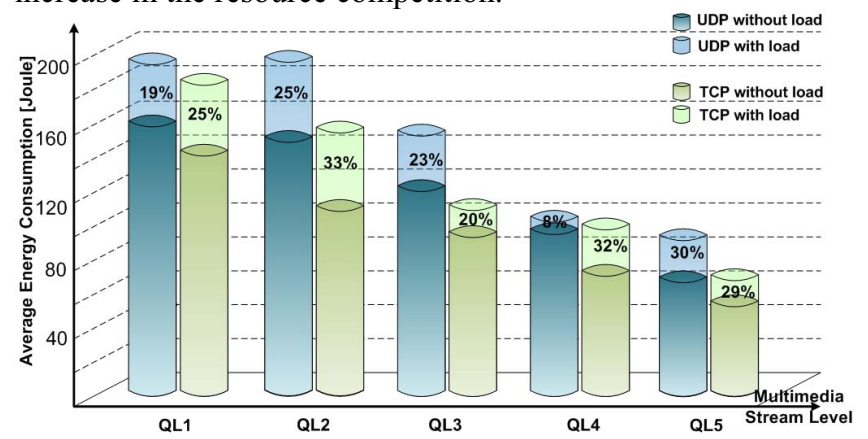

Figure 9. Scenario 1 vs. Scenario 3 Avg. Energy Consumption (UDP\&TCP) over the wireless interface

Even though the network load affects energy consumption for TCP video streaming (compared to Scenario 1); TCP is still more energy efficient than UDP. Also in this scenario the user perceived quality is not affected by the network load, the playback being smooth without interruptions.

7) Impact of Link Quality and Network Load on Energy Consumption

The impact of both link quality and network load can be studied by comparing Scenario 1 (where the mobile device is located near the AP without any background traffic) and Scenario 4 (where the device is located further away from AP with background traffic). In this case, both the link quality and the competition with the background traffic will impact the energy consumption. The Scenario 4 results are listed in Table VII. Even though there is a decrease in the overall channel traffic, there still is an increased number in total WLAN retransmissions.

An important parameter that needs to be mentioned is the Playout duration of the clip. Because of the competitive background traffic and the poor link quality, the mobile user will experience interruptions such as video freezing, leading to longer playback duration. This phenomenon has a more impact on the QL1 multimedia stream, resulting in long periods of buffer starvation and frequent 4-10s periods of video motion loss (the re-buffering periods represent almost $60 \%$ of the playout duration), while QL4 and QL5 are not affected.

TABLE VII. SCENARIO 4 - UDP AND TCP VOD

\begin{tabular}{|c|c|c|c|c|c|c|c|c|c|c|c|}
\hline & $\begin{array}{l}\text { Quality } \\
\text { Level }\end{array}$ & $\begin{array}{c}\text { Avg. } \\
\text { Energy } \\
{[\mathrm{J}]}\end{array}$ & $\begin{array}{l}\text { STDEV } \\
\text { Energy }\end{array}$ & $\begin{array}{l}\text { Avg. } \\
\text { Power } \\
\text { [mW] }\end{array}$ & $\begin{array}{c}\text { Dis- } \\
\text { charge } \\
{[\text { mAh] }}\end{array}$ & $\begin{array}{c}\text { Battery } \\
\text { Life } \\
{[\mathbf{h r s}]}\end{array}$ & $\begin{array}{c}\text { Avg. } \\
\text { Th. } \\
\text { [Mbps] }\end{array}$ & $\begin{array}{l}\text { Avg. } \\
\text { Ch. } \\
\text { Traffic } \\
{\left[\begin{array}{l}\text { Mbps }]\end{array}\right.}\end{array}$ & $\begin{array}{l}\text { Retr. } \\
{[\%]}\end{array}$ & $\begin{array}{c}\text { Play- } \\
\text { out } \\
\text { [s] }\end{array}$ & $\begin{array}{l}\text { Est. } \\
\text { MOS }\end{array}$ \\
\hline & QL1 & 1300 & 79.42 & 1362 & 98 & 3.62 & 1.32 & 20.13 & 11.83 & 958 & $<3$ \\
\hline & QL2 & 826 & 73.46 & 1193 & 62 & 4.13 & 1.02 & 20.71 & 10.35 & 695 & 3.58 \\
\hline & QL3 & 667 & 8.06 & 1015 & 50 & 4.86 & 0.45 & 20.15 & 9.12 & 659 & 3.43 \\
\hline & QL4 & 512 & 16.52 & 850 & 38 & 5.80 & 0.30 & 19.44 & 8.08 & 597 & 3.70 \\
\hline & QL5 & 468 & 4.52 & 783 & 35 & 6.29 & 0.14 & 18.88 & 11.75 & 597 & 3.38 \\
\hline & QL1 & 1283 & 136.68 & 1365 & 96 & 3.62 & 1.42 & 21.65 & 8.51 & 948 & $<3$ \\
\hline & QL2 & 784 & 35.92 & 1169 & 59 & 4.21 & 1.09 & 21.07 & 10.29 & 671 & 3.63 \\
\hline & QL3 & 596 & 112.34 & 966 & 45 & 5.0 & 0.69 & 21.31 & 10.45 & 617 & 4.03 \\
\hline & QL4 & 518 & 17.25 & 867 & 39 & 5.68 & 0.26 & 19.48 & 10.12 & 597 & 3.70 \\
\hline & QL5 & 456 & 4.08 & 763 & 34 & 6.45 & 0.16 & \begin{tabular}{|l|}
19.92 \\
\end{tabular} & \begin{tabular}{|l|}
9.75 \\
\end{tabular} & 597 & 3.38 \\
\hline
\end{tabular}

The impact of the re-buffering periods on the user perceived quality was assessed by the estimated MOS, which decreases 
with the increase in buffering percentage level, as explained in [14]. $15 \%$ buffering determines a quality decrease of 1 MOS unit and $60 \%$ buffering severely affects the quality with a corresponding drop of more than 1.5 MOS units. In both the case of UDP and TCP, our QL1 MOS will drop below the MOS of QL4 and QL5 (which maintain the same MOS as they do not introduce any buffering periods).

8) Impact of an Overloaded Network on Energy Consumption

In order to study what is happening when the network is overloaded, we increase the overall traffic so that the network is used at its maximum capacity. First we found the maximum capacity of this network by generating a UDP stream of $50 \mathrm{Mbps}$ (the maximum theoretical rate of an IEEE $802.11 \mathrm{~g}$ network is $54 \mathrm{Mbps}$ ). The average throughput of the stream reached 29-30Mbps. Based on this value we created background traffic using the mix from Table I. We ran Scenario 3 and 4 again, this time with 29-30Mbps of background traffic. The results for the two scenarios are presented in Table VIII.

It can be seen that even though the user is located near the AP he/she will experience interruptions with long periods of buffering, which was not the case when the network was loaded at 20-21Mbps. Moreover, when the user is located far from the AP, the QL1 streaming experience will be even worse, as the playout duration will reach almost nine times the normal playout length. In both cases, QL4 and QL5 are the most efficient in terms of playout duration and energy efficiency. In conclusion, in the case of an overloaded network, adapting the video quality to a lower level proves to be more efficient in terms of energy and user perceived quality.

TABLE VIII. OVERLOADED NETWORK - UDP AND TCP VOD

\begin{tabular}{|c|c|c|c|c|c|c|c|c|c|c|c|}
\hline & & $\mathbf{Q L}$ & $\begin{array}{c}\text { Avg. } \\
\text { Energy } \\
{[J]}\end{array}$ & $\begin{array}{l}\text { STDEV } \\
\text { Energy }\end{array}$ & $\begin{array}{l}\text { Avg. } \\
\text { Power } \\
\text { [mW] }\end{array}$ & $\begin{array}{c}\text { Dis- } \\
\text { charge } \\
{[\mathrm{mAh}]}\end{array}$ & $\begin{array}{c}\text { Battery } \\
\text { Life } \\
\text { [hrs] }\end{array}$ & $\begin{array}{c}\text { Avg. } \\
\text { Th. } \\
\text { [Mbps] }\end{array}$ & $\begin{array}{l}\text { Avg. } \\
\text { Ch. } \\
\text { Traffic } \\
\text { [Mbps] } \\
\end{array}$ & $\begin{array}{l}\text { Retr. } \\
{[\%]}\end{array}$ & $\begin{array}{c}\text { Playout } \\
{[\mathbf{s}]}\end{array}$ \\
\hline \multirow{10}{*}{$\mid \begin{array}{l}\bar{a} \\
\vdots \\
\bar{z} \\
z\end{array}$} & \multirow{5}{*}{$\overline{\mathrm{o}}$} & QL1 & 1308 & 158.61 & 1332 & 98 & 3.71 & 1.41 & 25.98 & 5.85 & 993 \\
\hline & & QL2 & 906 & 127.82 & 1113 & 68 & & 0.8 & 24.94 & 5.17 & 820 \\
\hline & & QL3 & 689 & 68.91 & 989 & 52 & & & & 3.48 & 704 \\
\hline & & QL4 & 518 & 7.4 & 866 & 39 & 5.68 & 0.34 & 26.50 & 3.43 & 597 \\
\hline & & QL5 & 461 & 7.38 & 774 & 35 & 6.36 & 0.16 & 24.76 & 5.86 & 597 \\
\hline & & QL1 & 1228 & 103.25 & 1358 & 92 & 3.63 & 1.37 & 26.65 & 4.33 & 909 \\
\hline & & QL2 & 833 & 189.2 & 1111 & 63 & 4.45 & 0.9 & 25.84 & 4.57 & 765 \\
\hline & & QL3 & 666 & 88.84 & 993 & 50 & 4.96 & 0.49 & 24.69 & 7.97 & 671 \\
\hline & & QL4 & 490 & 13.32 & 823 & 37 & 5.98 & 0.35 & 26.52 & 4.64 & 597 \\
\hline & & QL5 & 434 & 2.49 & 727 & 33 & 6.77 & 0.24 & 27.26 & 4.5 & 597 \\
\hline \multirow{10}{*}{ 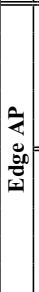 } & \multirow{5}{*}{ 容 } & QL1 & 4251 & 296.3 & 82 & 319 & 98 & 0.1 & 21.46 & 10.48 & 5165 \\
\hline & & QL2 & 1631 & 145.24 & 910 & 122 & 5.41 & 0.34 & 24.56 & 10.45 & 1793 \\
\hline & & QL3 & 789 & 32.36 & 1022 & 59 & 4.82 & 0.44 & 23.89 & 9.55 & 773 \\
\hline & & QL4 & 679 & 88.94 & 962 & 51 & 5.11 & 0.32 & 23.84 & 9.61 & 705 \\
\hline & & QL5 & 562 & 16.84 & 874 & 42 & 5.63 & 0.19 & 23.34 & 8.67 & 643 \\
\hline & \multirow{5}{*}{ ठิ } & QL1 & 44034 & 284.03 & 809 & 303 & 6.08 & 0.21 & 20.39 & 9.48 & 49987 \\
\hline & & QL2 & 1471 & 196.3 & 901 & 110 & 5.46 & 0.4 & 22.78 & 8.4 & 1633 \\
\hline & & QL3 & 751 & 95.12 & 974 & 56 & 5.06 & 0.4 & 24.14 & 9.32 & 773 \\
\hline & & QL4 & 518 & 25.06 & 867 & 39 & 5.78 & 0.31 & 24.79 & 9.86 & 619 \\
\hline & & QL5 & 456 & 17.51 & 763 & 34 & 6.46 & 0.16 & 24.24 & 8.89 & 611 \\
\hline
\end{tabular}

9) User Perceived Impact of Video Buffering on Multimedia Quality.

The subjective study did not aim to assess the impact of video buffering on user perceived quality. However, in order to have an idea of the users' perception of buffering, the test subjects were asked to rate (on a 1-5 scale) what they consider to be the MOS given different freeze periods $(<30 \mathrm{~s}, 1 \mathrm{~min}$, $2 \mathrm{~min}, 4 \mathrm{~min}$, and $>6 \mathrm{~min}$ ) for a 10 minute high-quality mobile video clip. The results illustrated in Fig. 10 show that, in order for the clip to maintain a Good quality level, the buffering time should not exceed 1 minute. Looking at the answers and abstracting other factors that may occur in a real streaming scenario, an excellent video quality (e.g., QL1) will have a similar quality, as perceived by the user, with QL5 (Fair), if the buffering time is equal or higher than two minutes. Switching to a lower quality level reduces the probability of re-buffering periods, thus avoiding the increase in playout duration, leading to energy savings and increase in user perceived quality.

\section{CONCLUSIONS}

This paper presents and in-depth study on how the wireless environment (e.g., link quality, network load) can impact the energy consumption of a mobile device while performing VoD. The tests were conducted on an Android Mobile Device in a controlled wireless (IEEE 802.11g) environment in order to better understand the impact of each parameter on the energy consumption. Five different quality levels of the multimedia stream were considered and their impact on the energy consumption was also analyzed. The results show that by changing the quality level of the multimedia stream the energy can be greatly saved while the user perceived quality level is still acceptable. This demonstrates the benefits that can be obtained by using an adaptive multimedia mechanism in terms of energy consumption. These mechanisms could be further improved in order to consider the energy consumption, making them even more energy efficient. Another important observation is the impact of the transport protocol (e.g., UDP, TCP) on the energy consumption. The results show that TCP is more energy efficient than UDP in all situations. After analyzing the Wireshark trace files, it has been noticed that TCP has a larger packet size distribution than UDP. This means that in case of TCP, less data is transmitted which leads to a decrease in energy consumption of the mobile device.

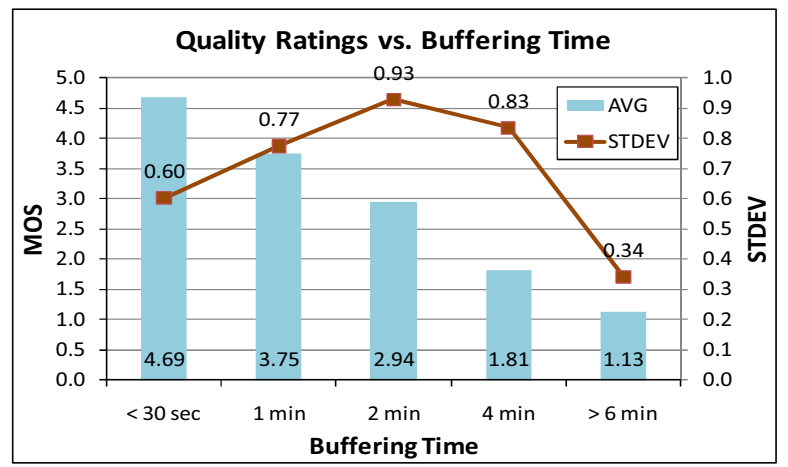

Figure 10. Users perception on video buffering impact on multimedia quality

Nowadays, user mobility can be highly predicted and together with the users' patterns of usage it can be possible to forecast where and when some wireless resources may be in high demand. Knowing the contextual information (e.g., link quality, network load, transport protocol, adaptive mechanism) and its impact on the energy consumption can help to make more energy-efficient use of the wireless resources. Thus, our findings demonstrate the necessity of considering networkrelated parameters when designing energy-efficient wireless video transmission schemes. 


\section{REFERENCES}

[1] Cisco Systems, Inc., "Cisco Visual Networking Index: Global Mobile Data Traffic Forecast Update, 2010-2015,” 2011.

[2] C. H. Muntean, "Improving learner quality of experience by content adaptation based on network conditions," Computers in Human Behavior, vol. 24, no. 4, pp. 1452-1472, 2008.

[3] M. Kennedy, H. Venkataraman, and G.-M. Muntean, "Dynamic stream control for energy efficient video streaming," in IEEE International Symposium on Broadband Multimedia Systems and Broadcasting (BMSB), 2011.

[4] ChangeWave Research, "New Smart Phone Owners Tell Us What They Really Think," 2010.

[5] J. Zhang et al., "Power-Aware Mobile Multimedia: a Survey (Invited Paper)," Journal of Communications 4, no.9, 2009.

[6] A. Rice and S. Hay, "Decomposing power measurements for mobile devices," in IEEE International Conference on Pervasive Computing and Communications (PerCom), 2010.

[7] N. Rodriguez, P. Hui, J. Crowcroft, and A. Rice, "Exhausting battery statistics: understanding the energy demands on mobile handsets," in Proc. of the $2^{\text {nd }}$ ACM SIGCOMM Workshop on Networking, Systems, and Applications on Mobile Handhelds, (MobiHeld), 2010.

[8] A. Rahmati and Lin Zhong, "Context-Based Network Estimation for Energy-Efficient Ubiquitous Wireless Connectivity," IEEE Transactions on Mobile Computing, vol. 10, no. 1, pp. 54-66, 2011.

[9] Yu Xiao, R. S. Kalyanaraman, and A. Yla-Jaaski, "Energy Consumption of Mobile YouTube: Quantitative Measurement and Analysis," in The Second International Conference on Next Generation Mobile Applications, Services and Technologies ( NGMAST), 2008.

[10] G.Perrucci, F.Fitzek, G.Sasso, and M.Katz, "Energy Saving Strategies for Mobile Devices using Wake-up Signals", Proc. ACM Press, 2008.

[11] V. Namboodiri and Lixin Gao, "Energy-Efficient VoIP over Wireless LANs," IEEE Transactions on Mobile Computing, vol. 9, no. 4, pp. 566581, Apr. 2010.

[12] ITU-T, P.910, "Subjective video quality assessment methods for multimedia applications" International Telecommunication Union, 2008.

[13] Canalys, "Press release: Android takes almost 50\% share of worldwide smart phone market", 2011.

[14] X. Tan et al., "Perceived Video Streaming Quality under Initial Buffering and Rebuffering Degradations", MESAQIN, 2006. 